\title{
Numerical reconstruction of digital holograms for conventional 3D display
}

\author{
Tomi Pitkäaho \\ RFMedia Laboratory \\ University of Oulu \\ Ylivieska, Finland \\ tomi.pitkaaho@oulu.fi
}

\author{
Thomas J. Naughton \\ Department of Computer Science \\ National University of Ireland \\ Maynooth, Ireland and \\ RFMedia Laboratory \\ University of Oulu \\ Ylivieska, Finland \\ tomn@nuim.cs.ie
}

\begin{abstract}
True hologram video displays are currently under development, but are not yet available. Because of this restriction, conventional 3D displays can be used with digital holographic data. However when using conventional 3D displays, holographic data has to be processed correctly to meet the requirements of the display. A unique property of digital holograms, namely that a single hologram encodes multiple perspectives, can be used to achieve this goal. Reconstructions from digital holograms at different perspectives are processed further to meet the requirements of the conventional 3D display, which are typically based on stereoscopic images of the scene.
\end{abstract}

\section{INTRODUCTION}

Holography is an imaging technique invented by Dennis Gabor in 1947, for which he got the Nobel prize in 1971 [1]. With holography it is possible to record and reconstruct realworld three-dimensional (3D) objects. A hologram is an image formed by interfering a laser beam with itself. This interference pattern can be recorded on a photographic plate, which requires chemical processing in the same manner as in conventional photography. Recent technical advancements have made it possible to replace this recording medium with a digital sensor like CCD (charged coupled device) or CMOS (Complementary metal-oxide-semiconductor), which are the same sensors used in digital photography. By using digital sensors we can talk about digital holography [2]. With digital holography both macroscopic and microscopic real-world 3D objects can be recorded [3-18]. The first commercial products, which are using digital holography, are from the field of 3D microscopy. Like conventional holography, digital holography also requires two different phases: recording and reconstruction. The reconstruction phase can be done numerically or optically. With a single capture of an object it is possible to extract $3 \mathrm{D}$ information encoded in a hologram. It is possible to extract multiple perspectives of the object from a single hologram. Also focusing on different depths is possible at the reconstruction phase. These two principles make holography a notable $3 \mathrm{D}$ imaging technique.

At this stage holographic displays are under research but actual holographic displays are not commercially available. This paper describes how conventional 3D displays can be used with holographic data. Holographic data for conventional $3 \mathrm{D}$ displays needs to be modified and processed before displaying. The unique properties of digital holograms can be used to obtain this.

Section 2 describes how a digital hologram's encoded perspectives can be used in data preparation. Section 3 describes different conventional 3D displays, their requirements for input data and how holographic data has to be processed to meet those requirements.

\section{DigITAL HOLOGRAMS'S ENCODED PERSPECTIVES}

Digital holograms can be reconstructed from different perspectives because of the way they are recorded. A single hologram encodes multiple perspectives of the scene, which is unique character of a hologram. After recording the hologram, in the reconstruction process different parts of a hologram can be used to reconstruct a scene from different perspective. When hologram center is located at 0,0 two different subsets of a hologram can be get as follows

$$
\Lambda[H(x, y)]=\left\{\begin{array}{l}
0, \text { if } x \geq 0 \\
H(x, y) \text { otherwise }
\end{array}\right.
$$

and

$$
\mathrm{P}[H(x, y)]=\left\{\begin{array}{l}
0, \text { if } x<0 \\
H(x, y) \text { otherwise }
\end{array}\right.
$$

From these subsets of a whole hologram the perspectives can be reconstructed as follows

$$
L=F_{\Lambda, z}
$$

and

$$
R=F_{\mathrm{P}, z}
$$

where $F$ is the Fresnel approximation at any distance given by

$$
F_{H, z}(x, y)=\left|\frac{-i}{\lambda z} H(x, y) \bullet \exp \left[i \pi \frac{x^{2}+y^{2}}{\lambda z}\right]\right|^{2}
$$

where the full hologram $H$ could be replaced by $\Lambda_{\text {or }} \mathrm{P} . \lambda$ is a wavelength of the light and $\bullet$ denotes a convolution operation. 
When using $\Lambda$ and $\mathrm{P}$, the equations above show how to get two horizontal perspectives. In that case half of the hologram data is used for each perspective (Figures 1a and 1b). To get multiple perspectives we can use different parts of the hologram data.

For a single view display a hologram reconstruction from one perspective is enough. When using digital hologram reconstructions as an input, this can be straight perspective of the scene and whole hologram data can be used. For the correct $z$ this is

$$
D(x, y)=F_{H, z}(x, y)
$$

where $\mathrm{D}$ is the display in question. This is the case for the conventional 2D displays.

For the stereoscopic displays requirement for the input data is two different perspectives of the scene. These perspectives can be reconstructed for the correct $z$ as follows

$$
\begin{gathered}
D_{0}=F_{\Lambda, z} \\
D_{1}=F_{\mathrm{P}, z}
\end{gathered}
$$

where $D_{0}$ and ${ }^{D_{1}}$ are interlaced, in left-right mode, or overunder mode as appropriate for the display in question.

For multi-view displays multiple perspectives of the scene are usually required. Multiple perspectives can be reconstructed from a digital hologram for the correct $z$ as follows

$$
\begin{gathered}
D_{0}=F_{\Lambda, z} \\
D_{1}=F_{\mathrm{P}, z} \\
D_{N}=F_{\mathrm{P}, z}
\end{gathered}
$$

where $D_{0}, D_{1}$ and $D_{N}$ are interlaced, in left-right mode, or over-under mode as appropriate for the display in question.

Amount of reconstructions is dependent of the display.

\section{CONVENTIONAL 3D DISPLAYS}

In an anaglyph two different images, each one of them representing different perspective of the scene, are combined to a one image. Observer gets three dimensional effect because one eye sees image only from one perspective. If observer is wearing red-green glasses, the eye covered with the red filter sees the long wavelengths i.e. red but not green and blue, and the eye covered with the green filter sees shorter wavelengths i.e. green and blue but not red. The brain combines these two different images together and interprets the differences to be depth difference. In a colour display an anaglyph image can therefore be presented so that one eye image is made with red and the other eye image with green. Most likely, there is a luminance difference between the two images. However, because of the rather quick photopic (cone vision) light adaptation of the retina the eye automatically adapts to any luminance difference between the eyes quite rapidly. Big benefit of this technique is that multiple persons wearing same kind of filtered glasses can get three-dimensional impression of the scene. Downside of this approach is that colours cannot be seen properly.

After reconstructing hologram from two different perspectives by using equations 1 - 5 speckle filtering (for example Fourier filtering) is applied to the reconstruction. How digital hologram reconstructions are used to make anaglyphs is dependent of the filters on the glasses. First we define an empty array $A(x, y, c)$ with same size than reconstruction, $c$ is the number of channels. If one is using three different channels (RGB) it would be 3. R stands for red, G for green and B for blue channel. Depending of the filters on the glasses $A$ gets different values. If glasses have a red filter in the left eye lens and a green filter in the right eye lens the process is

$$
\begin{gathered}
A(x, y, 1)=L(x, y) \\
A(x, y, 2)=R(x, y) \\
A(x, y, 3)=0
\end{gathered}
$$

Now with the left eye observer can see only the left reconstruction of the scene and with the right eye observer can see only the right reconstruction of the scene (Figure 1). Same procedure can be used with differently coloured lenses. In the data processing reconstructions from the left and right perspective has to be put in the correct colour channel.

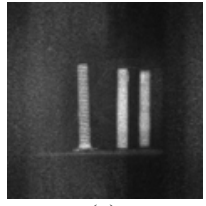

(a)

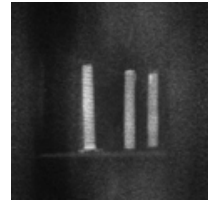

(b)

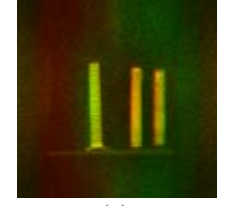

(c)
Figure 1. Speckle filtered reconstructions from two different perspectives (a) intensity of the left perspective reconstruction, (b) intensity of the right perspective reconstruction, and (c) anaglyph made from (a) and (b)

Shutter glasses are special glasses used with display to give $3 \mathrm{D}$ impression to the viewer. Shutter glasses contain LCD and polarizing filter. With this polarizer LCD becomes dark over one eye while other LCD is transparent and image of the display can be seen. In synchronisation with the refresh rate of the screen different perspectives are shown to the viewer wearing the glasses. Synchronisation can be done by using different radio architectures or infra red. Process to prepare holographic data for shutter glass display is following

$$
D(x, y, t)=\left\{\begin{array}{l}
L(x, y, t) \text { if } t \text { is even } \\
R(x, y, t) \text { otherwise }
\end{array}\right.
$$

where $x$ and $y$ are pixels. $t$ denotes a time.

Mirror stereoscope displays are most precise and versatile type of stereoscope for visual perception research purposes (Figure 2). With these displays it is guaranteed that right eye of the observer receives only the image meant for right eye and vice versa for the left eye of the observer. This kind of displays include different amount of mirrors and displays depending of the setup. Observer views the scene through mirrors where image from the display(s) is reflected.

To get accurate visual perception research results, observer has to be positioned accurately to make sure that each eye gets 
correct image. One possibility is to use chin rest in front of the mirror apparatus. This restricts also mobility of these displays because building this kind of apparatus is arduous and timeconsuming.

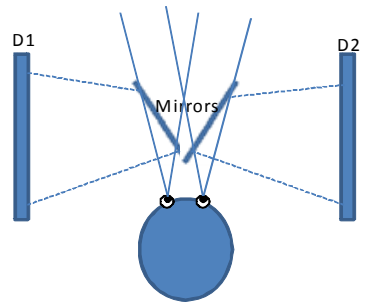

Figure 2. Example of mirror stereoscope display, Wheatstone apparatus. Observers sees two different images from displays D1 and D2, which are multiplexed with mirrors to one image

Procedure to process holographic data for mirror display is as follows

$$
D_{0}(x, y)=L(-x,-y)
$$

and

$$
D_{1}(x, y)=R(-x,-y)
$$

for the two displays, where $D_{0}, D_{1}$ are on the left and right sides of the viewer, respectively. If apparatus includes odd number of mirrors negative values are needed.

\section{CONCLUSIONS}

We have shown that a single digital hologram can be used with conventional 3D displays. However holographic data has to be processed to meet the requirements of the display and data processing is display-specific. We have described data preparation for different kind of conventional 3D displays. Other conventional 3D displays do exists but they are not covered here. They are left out of this because they are usually using the same principles than the displays described in this paper. The basic principle in the displays is that of a stereoscopic image pair, which gives the $3 \mathrm{D}$ impression to the observer when viewed appropriately. Also displays with multiple perspective view requirement can be implemented with digital holograms. Digital holograms are suitable for conventional 3D displays as long as holograms encode different perspectives.

\section{ACKNOWLEDGMENT}

This research received funding from Science Foundation Ireland under the National Development Plan, Academy of Finland, and the European Community's Seventh Framework Programme FP7/2007/2013 under grant agreement no. 216105 ("Real 3D").

\section{REFERENCES}

[1] D. Gabor, "A new microscopic principle," Nature, vol. 161, pp. 777-778, 1948.

[2] U. Schnars and W. P. Juptner, "Direct recording of holograms by a CCD target and numerical reconstruction," Appl. Opt., vol. 33, pp. 179-181, Jan. 1994.

[3] J. W. Goodman and R. W. Lawrence, "Digital image formation from electronically detected holograms," Appl. Physics Lett., vol. 11, pp. 77-79, 1967.

[4] M. A. Kronrod, N. S. Merzlyakov, and L. P. Yaroslavskii, "Reconstruction of a hologram with a computer," Soviet Physics Technical Physics, vol. 17, pp.333, 1972.

[5] J. H. Bruning, D. R. Herriott, J. E. Gallagher, D. P. Rosenfeld, A. D. White, and D. J. Brangaccio, "Digital wavefront measuring interferometer for testing optical surfaces and lenses," Appl. Opt., vol. 13, pp. 2693-2703, 1974.

[6] L. Onural and P. D. Scott, "Digital decoding of in-line holograms," Opt. Eng., vol. 26, pp. 1124-1132, 1987.

[7] I. Yamaguchi and T. Zhang, "Phase-shifting digital holography," Opt. Lett., vol. 22, pp 1268-1270, 1997.

[8] E. Cuche, P. Marquet, and C. Depeursinge, "Simultaneous amplitude-contrast and quantitative phase-contrast microscopy by numerical reconstruction of Fresnel off-axis holograms," Appl. Opt., vol. 38, pp. 6994-7001, 1999.

[9] G. Pedrini, P. Froning, H. Tiziani, and F. Santoyo, "Shape measurement of microscopic structures using digital holograms," Opt. Commun., vol. 164, pp. 257-268, 1999.

[10] T.J. Naughton, Y. Frauel, B. Javidi, and E. Tajahuerce, "Compression of digital holograms for three-dimensional object reconstruction and recognition," Appl. Opt., vol. 41, pp. 41244132, 2002.

[11] P. Ferraro, G. Coppola, S. Nicola, A. Finizio, and G. Peirattini, "Digital holographic microscope with automatic focus tracking by detecting sample displacement in real time," Opt. Lett., vol. 28, pp. 1257-1259, 2003.

[12] C. Mann, L. Yu, C. Lo, and M. Kim, "High-resolution quantitative phase-contrast microscopy by digital holography," Opt. Express, vol. 13, pp. 8693-8698, 2005.

[13] J. Garcia-Sucerquia, W. Xu, S. K. Jericho, P. Klages, M. H. Jericho, and H.J. Kreuzer, "Digital in-line holographic microscopy," Appl. Opt., vol. 45, pp. 836-850, 2006.

[14] Y. Frauel, T.J. Naughton, O. Matoba, E. Tajahuerce, and B. Javidi, "Three-dimensional imaging and processing using computational holographic imaging," Proc. IEEE, vol. 94, pp. 636-653, 2006.

[15] F. Dubois, C. Schockaert, N. Callens, and C. Yourassowsky, "Focus plane detection criteria in digital holography microscopy," Opt. Express, vol. 14, pp. 5895-5908, 2006.

[16] B. Javidi, S. Yeom, I. Moon, and M. Daneshpanah, "Real-time automated 3d sensing, detection, and recognition of dynamic biological micro-organic events," Opt. Express, vol. 14, no. 9, pp. 3806-3829, 2006.

[17] O. C. Chee, V. R. Singh, E. Sim, and A. K. Asundi, "Development of a simple user-friendly commercial digital holographic microscope," Proc. SPIE 6912, 69120V, 2008.

[18] M. Kempkes, E. Darakis, T. Khanam, A. Rajendran, V. Kariwala, M. Mazzotti, T.J. Naughton, and A.K. Asundi, "Three dimensional digital holographic profiling of micro-fibers," Opt. Express, vol. 17, pp. 2938-2943, 2009. 